\title{
LUCERNA ROMANA DE BRONCE PROCEDENTE DE CASTRO URDIALES (CANTABRIA)
}

\author{
POR \\ JOSÉ LUIS PÉREZ SÁNCHEZ Y ALICIA RUIZ GUTIÉRREZ \\ Arqueólogos - Universidad de Cantabria *
}

\section{RESUMEN}

En el artículo se da a conocer una lucerna de bronce romana recuperada en una excavación de urgencia realizada en Castro Urdiales. La pieza corresponde al tipo Loeschcke XX, bien representado en los yacimientos de Pompeya y Herculano; se fabricó en la segunda mitad del siglo I y primeros años del II, y tuvo una amplia difusión geográfica por distintas provincias del Imperio. El ejemplar objeto de este estudio apareció en un contexto arqueológico que puede relacionarse con la colonia Flaviobriga citada en las fuentes clásicas.

\section{SUMMARY}

This article is intended to present a Roman lamp of bronze, which was obtained from a rescue excavation in Castro Urdiales (Cantabria). This piece can be identified with the type known as Loeschcke XX, very well represented in the sites of Pompeii and Herculaneum, dated to the second half of the first century and the beginning of the second century AD. It was widespread through the different provinces of the Empire. The piece under study appeared in an archaeological context that has been related to the colonia Flaviobriga; the latter is mentioned by the classic sources.

La localidad de Castro Urdiales, capital del municipio del mismo nombre en la costa oriental de Cantabria, ha sido escenario de varios trabajos arqueológicos en los últimos años, que han permitido ampliar nuestros conocimientos sobre su pasado romano. Dos excavaciones de urgencia en la calle Ardigales han dejado a la luz los restos de una edificación, al parecer privada, de época flavia, correspondiente según todos los indicios a la ciudad romana de Flaviobriga, recogida en las obras de Plinio, quien la cita como colonia (Nat. Hist. IV, 110) y Ptolomeo (Geogr. II, 6, 7). Los resultados de estas excavaciones y de otras actuaciones arqueológicas en el casco histórico de la ciudad, realizadas entre los años 1991 y 1994, han sido publicados recientemente en un libro editado por el Ayuntamiento de

* Agradecemos a Luis Teira Mayolini el dibujo de la pieza.
Castro Urdiales '. En esta obra se anuncia la publicación independiente de una pieza arqueológica que, por su singularidad, merece un estudio detallado; nos referimos a una lucerna de bronce de la que nos ocupamos en estas líneas, hallada durante la excavación de 1991 en la calle Ardigales ${ }^{2}$. En concreto, la lucerna apareció en el perfil oeste del Cuadro A del área excavada, en el interior de una de las estancias de la edificación flavia, a unos $1,40 \mathrm{~m}$ de profundidad, en un nivel de uso o abandono de la misma, que contenía numerosos fragmentos de TSH y cerámica común, datables en la época flavia y el siglo II. Debido a las limitaciones de la excavación, no podemos determinar si esta edificación romana, de la que pudieron excavarse tan sólo parcialmente tres estancias y restos de un posible patio, correspondía a una domus aislada o, como parece más probable, formaba parte de una manzana integrada en el entramado de la ciudad; en cualquier caso, su carácter privado o doméstico parece seguro. En la estancia en la que apareció la lucerna no se conservaban restos de pavimento, pero sí en la habitación colindante, de opus signinum, así como fragmentos de estucos desprendidos de los muros, con decoración pintada. En la actualidad, la lucerna se conserva en el Museo Regional de Prehistoria y Arqueología de Cantabria, en Santander ${ }^{3}$.

\section{DESCRIPCIÓN}

Se trata de una lucerna de bronce realizada a molde, de buena factura; el estado de conservación es bueno, si bien el asa se encuentra fragmentada, faltándole el remate decorativo que suelen presentar este tipo de ejemplares, por lo común consistente en un tema figurativo en relieve: máscara o cabeza de

\footnotetext{
1 Iglesias, J. M., y Ruiz, A. (dirs.), Flaviobriga. Castro Urdiales romano. Arqueología de intervención (años 19911994), Bilbao, 1995

2 Iglesias, J. M., y Ruiz, A., (dirs.), op. cit. (nt. 1), nº 94 , p. 50 , fig. 18 , lám. 11 .

${ }^{3}$ La lucerna lleva el no 293 en el inventario del depósito.
} 


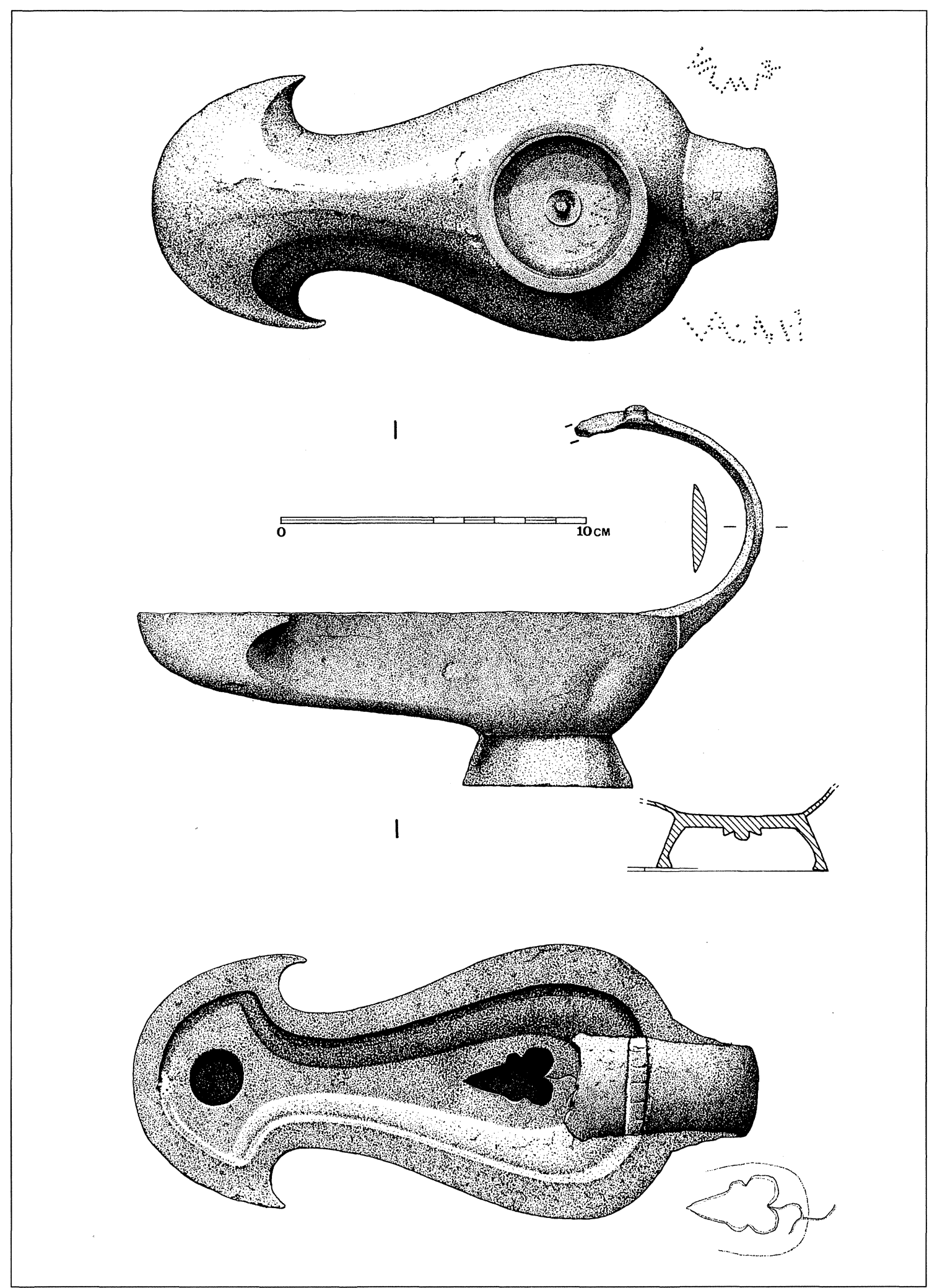

Fig. 1.-Lucerna con inscripción hallada en Castro Urdiales. 
animal (león, pantera, caballo, ganso, gallo...) ${ }^{4}$. Sus dimensiones máximas, incluyendo el asa, son: 20,5 $\mathrm{cm}$ de longitud, $9 \mathrm{~cm}$ de anchura y $12,5 \mathrm{~cm}$ de altura. El recipiente de la lámpara es piriforme, acabado en rostrum semicircular, de $18 \mathrm{~cm}$ de longitud, $9 \mathrm{~cm}$ de anchura y $5,7 \mathrm{~cm}$ de altura. Presenta la cara superior moldurada y el borde levantado con objeto de facilitar la tarea de relleno o alimentación de la lámpara. El orificio de iluminación en el rostrum es circular, de 1,6 cm de diámetro, en tanto que el de alimentación, en el centro del discus, tiene forma de hoja polilobulada, de $3 \mathrm{~cm}$ de longitud, acompañado de un trazo inciso a modo de tallo. La lucerna se apoya sobre un pie anular alto, de perfil cónico, que mide 5,7 $\mathrm{cm}$ de diámetro y $1,7 \mathrm{~cm}$ de altura. Del extremo opuesto a la piquera arranca el asa curva, de sección plano-convexa, de 3,2 cm de anchura; se aprecia claramente la unión de ésta al infundibulum o recipiente de la lámpara, siendo evidente que se trata de un elemento realizado de forma separada por el broncista, con un molde específico, al igual que el pie.

La lucerna presenta dos inscripciones, una de ellas ubicada en la cara externa de la base y la otra en el arranque del asa. Ambas están realizadas en escritura capital, con las letras grabadas por medio de puntos. La de la base, de apariencia más cuidada, presenta el texto VAL MAR, con la letra $A$ sin travesaño y enlace $A L$. En la inscripción del asa se lee $V A L . M R$, con nexo $V A L$, donde la letra $A$ lleva travesaño, y signo de interpunción antecediendo a las letras $M R$, de difícil lectura. Consideramos que las dos

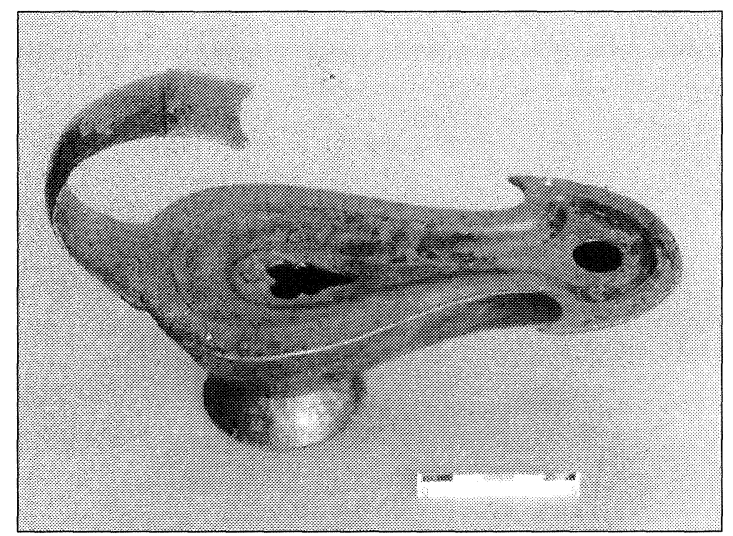

Fig. 2.-Vista superior de la lucerna.

inscripciones se refieren a un mismo nombre personal compuesto de dos elementos: VAL, que hemos de relacionar con el nomen latino Valerius/-a, y MAR,

\footnotetext{
${ }^{4}$ Loeschcke, S., Lampen aus Vindonissa. Ein Beitrag zur Geschichte von Vindonissa und des antiken Beleuchtungswesens, Zurich, 1919, p. 323.
}

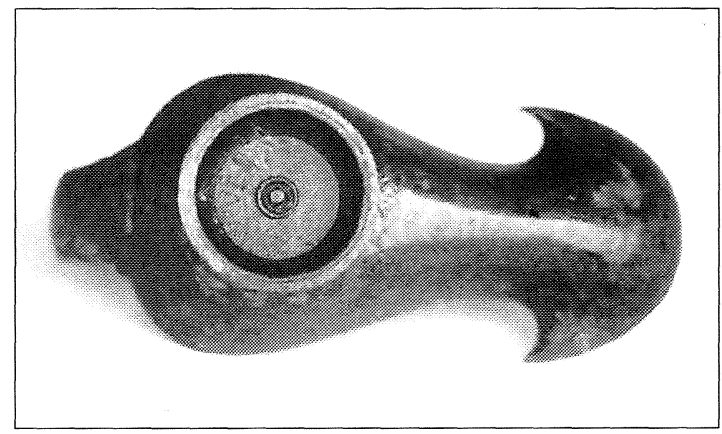

Fig. 3.-Vista inferior.

que puede corresponder a varios cognomina también latinos: Martialis, Marcellus/-a, Marcellinus/-a, Marcianus/-a, Marinus/-a, etc. No parece probable que estemos ante una marca de fábrica, sino más bien ante un grafito en el que se hace constar el nombre del propietario de la pieza.

\section{TIPOLOGÍA Y PARALELOS}

La lucerna que acabamos de describir corresponde a un tipo bien definido: Loeschcke XX/Iványi XXXIV, de origen helenístico, fabricado en la península itálica y muy difundido por diversas regiones del imperio romano. En la clasificación que realiza Loeschcke de las lámparas del campamento militar de Vindonissa (Suiza), el Tipo XX, junto con el XXI, queda englobado dentro de un grupo de lucernas de

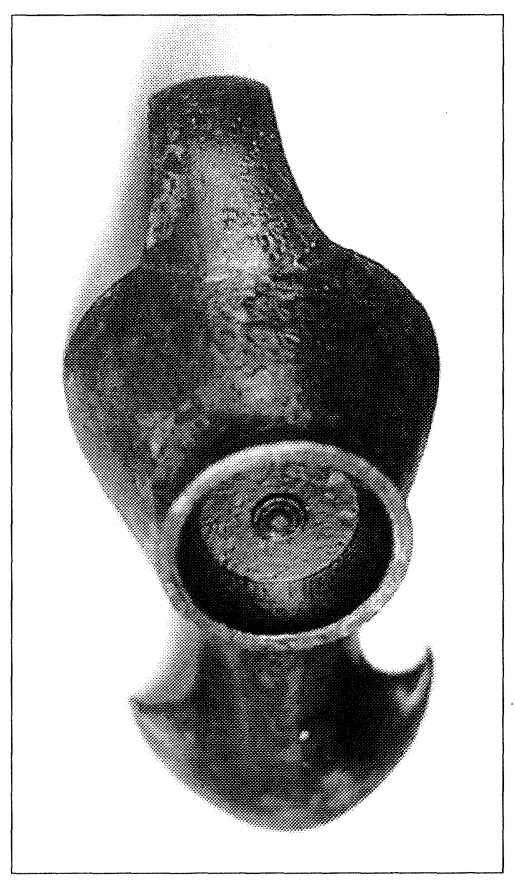

Fig. 4.-Detalle inferior. 
bronce caracterizadas por presentar cuerpo piriforme, o «con forma de pera» (birnenförmige Bronzenlampen). El primero, al que se adscribe nuestro ejemplar, corresponde a grandes lámparas piriformes, de rostrum anguloso; mientras que el segundo engloba lámparas piriformes con asa en forma de creciente lunar y arandelas de suspensión. Según Loeschcke, el Tipo XX se fabricó en la segunda mitad del siglo I y fue imitado en cerámica en el siglo II. Este autor indica su extraordinaria abundancia en Pompeya, de lo que se deduce que estaba de moda en el momento de destrucción de la ciudad, en el año $79^{5}$. Iványi, en su estudio sobre las lucernas de Panonia, equipara el tipo Loeschcke XX al Tipo XXXIV de su clasificación, donde agrupa lucernas a las que define por presentar cuerpo alargado, finalizado en rostrum semicircular, de aristas angulosas ${ }^{6}$.

Además de los ejemplares recopilados por los dos autores anteriores, que sirven de base a sus respectivas clasificaciones tipológicas, aún vigentes, podemos citar una larga lista de paralelos, de diversa procedencia geográfica, que testimonian la gran aceptación que tuvo este tipo de lucernas en varias provincias romanas. En primer lugar, destaca el importante conjunto hallado en Pompeya y Herculano ${ }^{7}$, en el que se apoya Loeschcke para establecer sus consideraciones cronológicas. A los ejemplares que proceden con seguridad de estos yacimientos itálicos, hemos de sumar los distribuidos por varios museos europeos y colecciones privadas, de los que se presume el mismo origen. Este es el caso de algunas lucernas del Museo Arqueológico Nacional de Madrid estudiadas por Blázquez ${ }^{8}$, procedentes de las antiguas colecciones de la Biblioteca Nacional y del Museo de Historia Natural.

${ }^{5}$ Loeschcke, S., op. cit. (nt. 4), pp. 323-325, no 1055, pp. 453-454, Taf. II y XXI, Abb. 37.

${ }^{6}$ Iványi, D., Die pannonischen Lampen. Eine typologisch-chronologische Übersicht, Dissertationes Pannonicae, Sér. 2, 2, Budapest, 1935, pp. 23 y 301, Taf. LXI,2, 5 y 6.

7 En el museo de Nápoles se conservan, según Valenza, unas 400 lucernas de bronce, en su mayor parte de Pompeya y Herculano, entre las que se encuentran varios ejemplares del tipo representado en Castro Urdiales (Valenza Mele, N., «Le lucerne di bronzo del Museo di Napoli», en VV.AA., L'instrumentum domesticum di Ercolano e Pompei nella prima età imperiale, Roma, 1977 a, pp. 157 y 160, Tav. LXXIV,12). Véase también: Valenza Mele, N., «Lucerne in bronzo da Pompei ed Ercolano. Classificazione e tipologia», Actes du VIe Colloque International sur les bronzes antiques (17-21 mai 1976), Lyon, 1977 b, p. 182.

${ }^{8}$ Blázquez, J. M., «Veintinueve lámparas romanas de bronce del MAN», Zephyrus, X, 1959, nº 1-3 y 28, pp. 159161 y 169 , láms. I, II y X. Estas lucernas se encuentran recopiladas en una obra anterior: Thouvenot, R., Catalogue des figurines et objets de bronze du Musée Archéologique de Madrid, I. Bronzes grecs et romains, París, 1927, $\mathrm{n}^{\circ} 412$, 413,421 y 441, pp. 80 y 82 .
Walters, en su catálogo de lámparas griegas y romanas del British Museum de Londres, englobó dentro de la Forma 6 diez ejemplares correspondientes al tipo Loeschcke XX/Iványi XXXIV, todos ellos de antiguas colecciones privadas y diversas procedencias geográficas ${ }^{9}$. En un estudio sobre las lámparas de bronce antiguas de la Dacia Superior, Baluta recopila dos piezas del mismo tipo, que tienen en común su tosquedad, datadas en la primera década del siglo II ${ }^{10}$. En la vecina provincia de Tracia, se encuentra otra lucerna, de calidad semejante a las dos anteriores de Dacia, conservada en el Museo Arqueológico Nacional de Sofía ${ }^{11}$. En la Europa central, podemos citar un ejemplar de Lauriacum (Austria) ${ }^{12}$, y en la Galia otro de Vienne ${ }^{13}$.

Dentro de la Península Ibérica, además de las piezas del Museo Arqueológico Nacional de Madrid, de presumible origen foráneo, se conservan otras halladas en suelo peninsular. Una de ellas, procedente de León ${ }^{14}$, presenta el asa decorada con una máscara; otra, con el mismo tema decorativo, fue hallada en la antigua Iptuci, en Villamartín (Cádiz) ${ }^{15}$. En la región del noroeste, existen también ejemplos, como el de Filloedo (Salvatierra de Miño, Pontevedra), con el asa rematada en cabeza de ánade ${ }^{16}$.

9 Walters, H. B., Catalogue of the Greek and Roman Lamps in the British Museum, Londres, 1914, $\mathrm{n}^{\circ}$ 84-85, pp. 14-16, Plates VI y VII.

10 Baluta, L. C., «Les lampes antiques en bronze de la Dacie Supérieure. Classification et Chronologie», Bronzes Héllénistiques et Romains. Tradition et Renouveau, Actes du $\mathrm{V}^{\mathrm{e}}$ Colloque International sur les bronzes antiques (Lausanne, 1978), Cahiers d'Archéologie Romande, $\mathrm{n}^{\circ}$ 17, Lausana, 1979 , p. 206, Pl. $116, \mathrm{n}^{\circ} 7$ y 10

11 Kuzmanov, G., Antike Lampen. Sammlung des Nationalen Archäologischen Museums, Sofía, 1992 (edición en búlgaro), $\mathrm{n}^{\circ} 423$, pp. 52-53.

12 Deringer, H., Römische Lampen aus Lauriacum, Linz, $1965, \mathrm{n}^{\circ} 403$, pp. 68 y 126 , Taf. XIV.

13 Se trata de una lucerna con máscara diademada en el asa, tema decorativo que Boucher considera de origen helenístico, y más exactamente alejandrino (Boucher, S., Recherches sur les bronzes figurés de Gaule pré-romaine et romaine, Roma, 1976, n 347, Pl. 71).

${ }_{14}$ Gómez Moreno, M., Catálogo monumental de España. Provincia de León, Madrid, 1926, p. 50, lám. X; Mañanes, T., «Bronces romanos en la provincia de León», Homenaje al Prof. Martín Almagro Basch, III, Madrid, 1983, pp. 399410; VV.AA., Los Bronces romanos en España, Madrid, $1990, n^{\circ} 206$, p. 272; VV.AA., Astures. Pueblos y culturas en la frontera del Imperio Romano, Gijón, 1995, p. 285.

15 Romero, E., Catálogo monumental de España: Provincia de Cádiz, Madrid, 1934, lám. LXXV; Los Bronces, op. cit. (nt. 14), n' 207, p. 273.

16 Acuña Castroviejo, F., «La cultura en la Galicia romana», en VV.AA., La Romanización de Galicia, La Coruña, 1992 ( $1^{\text {a }}$ ed. 1976), p. 70, lám. sin nº; Filgueira Valverde, F., y García Alen, A., Materiales para la Carta Arqueológica de la Provincia de Pontevedra, Pontevedra, 1955, p. 178; Los Bronces, op. cit. (nt. 14), no 210, p. 274. 


\section{ORIGEN Y CRONOLOGÍA}

En general, la datación de las lucernas de bronce resulta problemática y ha de abordarse con cautela, debido a varios factores. Por un lado, hay que tener en cuenta que se trata de piezas poco abundantes, minoritarias en comparación con las lucernas fabricadas en cerámica, debido a su coste y a la costumbre que desde antiguo se ha tenido de fundir los objetos de bronce inservibles para reaprovechar el metal ${ }^{17}$. La escasez de ejemplares llegados a nosotros limita las posibilidades de estudio, sobre todo a nivel regional, y dificulta la determinación de unos márgenes cronológicos fiables para cada tipo de lucerna. Por otro lado, cabe pensar que esta clase de objetos tendría una larga prolongación de uso, sin duda mucho mayor que la de los ejemplares de cerámica. Otro de los problemas para fijar una datación es la escasez de lucernas de bronce procedentes de contextos arqueológicos bien documentados, pues tradicionalmente han circulado en manos de anticuarios y coleccionistas particulares. En efecto, muchas de las lucernas de bronce depositadas en los museos carecen de referencias adecuadas sobre su procedencia estratigráfica o lugar concreto del hallazgo, al ser originarias de antiguas colecciones privadas o públicas pobremente documentadas. Las clasificaciones tipológicas, por ello, no deben considerarse un medio de datación infalible ${ }^{18}$, especialmente si están elaboradas a partir de los fondos de museos donde se agrupan piezas sin contexto arqueológico.

Los estudios clásicos, publicados desde principios de siglo, sobre las lucernas de algunos campamentos militares del limes, y especialmente de los yacimientos de Pompeya y Herculano, arrojan luz sobre el problema cronológico, pero si bien las conclusiones son especialmente válidas para las lucernas de cerámica, no son tan firmes para las de soporte metálico. Según Valenza, la mayor duración de uso de éstas impide formular una cronología precisa, incluso en el caso de Pompeya, pues, aunque este yacimiento aporta la datación ante quem del año 79, ignoramos hasta qué punto perduraron los tipos de lucerna que estaban en uso en el momento de su destrucción ${ }^{19}$.

A pesar de las limitaciones anotadas, la mayoría de los autores coincide en una datación bastante ajustada del tipo concreto de lucerna representado

17 Cicerón, Verr., IV, 25, 55; Valenza Mele, N., 1977 a, op. cit. (nt. 7), p. 157

18 Baluta, L. C., op. cit. (nt. 10), p. 205

19 Valenza Mele, N., 1977 a, op. cit. (nt. 7), p. 157. en Castro Urdiales, comprendida entre la segunda mitad del siglo I y los inicios del II. Loeschcke, uno de los primeros autores en pronunciarse sobre el tema, indicaba la ausencia de indicios sobre la existencia de este tipo de lámpara en la primera mitad del siglo I; y señalaba la fabricación de imitaciones en cerámica durante el siglo II, e incluso ya desde finales del siglo $\mathrm{I}^{20}$. Valenza es partidaria de una datación algo más amplia, que quizás deba remontarse al segundo cuarto del siglo I, pues algunos de los ejemplares de Pompeya, soterrados en la catástrofe del 79, presentan signos de un uso prolongado. Esta autora llama la atención sobre la corta perduración del modelo, pese a su amplia difusión geográfica, pudiendo afirmarse que no se encuentran piezas posteriores a la mitad del siglo $\mathrm{II}^{21}$.

En efecto, es posible que esta clase de lucernas de bronce se fabricara durante un período de tiempo reducido, así como también las imitaciones de cerámica ${ }^{22}$, que no debieron de tener gran éxito, debido quizás a las características del modelo ${ }^{23}$ y en concreto a la forma del asa, demasiado frágil para ser elaborada en ese material. Por otro lado, el estudio comparativo de los distintos ejemplares permite apreciar ciertas diferencias, tanto en aspectos formales como, especialmente, en la calidad de fabricación, las cuales pueden explicarse bien por una mayor perduración cronológica del modelo o por una diversificación de la producción en distintas provincias del Imperio.

Según Valenza, es prematuro indicar la localización del centro de producción itálico, e incluso determinar si éste fue único o bien existió una multiplicidad de áreas productoras, reflejada en la variedad de los tipos atestiguados. La misma autora señala que tal diversidad de tipos se hace menor en las provincias después del siglo I, coincidiendo con la desaparición de innovaciones, lo que refleja el nacimiento de una producción local en varias pro-

${ }^{20}$ Loeschcke, S., op. cit. (nt. 4), pp. 324-325. En esta obra sobre las lucernas de Vindonissa, se recoge un interesante ejemplar de cerámica, con el asa rematada en prótomo de caballo, sellado por el alfarero de «Firmalampen» Eucarpus, de finales del siglo I ( $\mathrm{n}^{\circ} 1080$, pp. 340-341, Abb. 22).

21 Valenza Mele, N., 1977 a, op. cit. (nt. 7), p. 160.

${ }^{22}$ La dependencia de los ejemplares de cerámica con respecto a los metálicos es muy evidente, como puede comprobarse en los de origen dacio publicados por Dora Iványi: Iványi, D., op. cit. (nt. 6), p. 305, Taf. XV, 1, 2 y 3. En la Península Ibérica, contamos con una lucerna de cerámica, conservada en el Museo de Mérida, que presenta el asa rematada por una cabeza de caballo, muy vinculada a las lucernas de bronce del tipo que nos ocupa, datada en el siglo I (Gil Farrés, O., «Interesante lucerna inédita del Museo de Mérida», AEspA, XXII, 1949, pp. 213-216).

23 Valenza Mele, N., 1977 b, op. cit. (nt. 7), p. 182. 
vincias del Imperio que absorbería todo el mercado interno ${ }^{24}$. El comercio de moldes facilitaría el surgimiento de estos talleres locales que, con mayor o menor éxito, imitaban los modelos itálicos.

Por el momento, ignoramos hasta qué punto las diferencias formales reflejadas en los ejemplares de la Península Ibérica puedan tener un significado cronológico, común al de otras provincias, o bien geográfico, resultado de una posible producción peninsular. En principio, es posible intuir una menor antigüedad o fidelidad al prototipo itálico en las lucernas que presentan recipiente de tendencia barquiforme, con la molduración de la cara superior del infundibulum simplificada y la base plana o pie reducido a su mínima expresión, como ocurre en la lucerna de Filloedo, datada en el siglo ${ }^{2}{ }^{25}$. El ejemplar de Castro Urdiales, en cambio, mucho más estilizado, de buenas proporciones y aristas bien perfiladas, constituye un artículo de considerable calidad técnica, que hemos de considerar itálico. Por sus características y contexto arqueológico es datable en la segunda mitad del siglo I, más concretamente en época flavia, en especial si apoyamos su relación con la posible llegada de pobladores a la colonia Flaviobriga fundada por Vespasiano. 